\title{
Three-Phase AC-to-AC Series-Resonant Power Converter with a Reduced Number of Thyristors
}

\author{
J. Ben Klaassens and Freddie de Beer
}

\begin{abstract}
Ac-ac series-resonant converters have been proven to be functional and useful. Power pulse modulation with internal frequencies of tens of $\mathbf{k H z}$ and suited for multikilowatt power levels is applied to a series-resonant converter system for generating synthesized multiphase bipolar waveforms with reversible power flow and low distortion. The high pulse frequency allows the application of the principle of modulation and demodulation for fast system response. The use of a seriesresonant circuit for power transfer and control obtains natural current commutation of the thyristors and the prevention of excessive stresses on components. Switches are required which have bidirectional current conduction and voltage blocking ability. The conventional series-resonant ac-ac converter applies a total for $\mathbf{2 4}$ anti-parallel thyristors. An alternative circuit configuration for the series-resonant ac-ac converter with only 12 thyristors is presented. Use of the converter results in a higher efficiency and lower costs. The alternative power circuit has three neutrals, related to the polyphase source, the load and the converter, which may be interconnected. If they are connected, the high-frequency component of the source and load currents will flow through the connection between the neutrals. The test results of a converter system generating threephase sinusoidal input and output waveforms have demonstrated the significant aspects of this type of power interfaces.
\end{abstract}

\section{INTRODUCTION}

A $\mathrm{N}$ ac-ac series-resonant conversion system is being developed because of its many potential applications, such as ac motordrives including the processes of the braking and reversing of the direction of rotation. Another possible application is the asynchronous coupling of two polyphase ac systems, such as interfacing a windmill generator and a power grid, in which a coupling element is required in the form of a power interface. The presented ac-ac series-resonant power converter shows good performance due to its intrinsic characteristics.

The series-resonant converter employs a modulated train of power pulses to generate a multiphase bipolar waveform. The high resolution obtained for the control and distribution of electric energy paves the way for this process of wave shaping. The fast response has its roots

Manuscript received March 30, 1989; revised September 1, 1990. This paper was presented at the 1989 IEEE Power Electronics Specialists Conference, Milwaukee, WI, June 26-29.

The authors are with Delft University of Technology, Laboratory of Power Electronics, Mekelweg 4, 2628 CD Delft, The Netherlands.

IEEE Log Number 9100538. in the high internal frequency and the applied philosophy of control which avoids the interposition of low frequency filters in its signal processing system.

A complex process of excitation of the double excited $L C$-circuit makes it possible to operate the power converter for both directions of the power flow. The natural limitation for the conversion ratio of the conventional series-resonant converter to be one, can be overcome. The developed converter system is studied with respect to the coupling of a three-phase ac voltage source and a three-phase ac load. But the electric energy can also be derived from a two terminal dc source of supply.

The use of a series-resonant circuit for power transfer and control, involves natural current commutation of the thyristors. Improved reliability results from the moderate stresses arising during the switching of semiconductor devices under zero current conditions in the resonant circuit.

The resonant circuit is considered to be a high-frequency alternating-current link between two sets of switching matrices at the input and output terminals of the ac-ac inverter. This high-frequency link allows the elimination of all low-frequency filters and transformers, which are a requirement by conventional ac-ac converters because of the interposition of a low-frequency directvoltage or direct-current link between two sets of switching matrices.

The generation of high-frequency components in the source and load currents can be reduced to relatively small quantities by the application of high-frequency filters of moderate size

Direct operation by an ac-current link requires power switches to have bidirectional blocking and conducting capabilities. Bidirectional current capability is also mandatory for bipolar source and load currents.

The more conventional series-resonant ac-ac converter applies, therefore, bipolar switches in arrangements of anti-parallel thyristors with a total of 24 individual thyristors as shown in Fig. 1(a) [2].

An alternative circuit configuration for the series-resonant ac-ac converter with only 12 thyristor switches as shown in Fig. 1(b), is presented. Simulation and experimental work have clearly demonstrated that the new converter topology has, in principle, the same potentials as the previous one. 
(a)

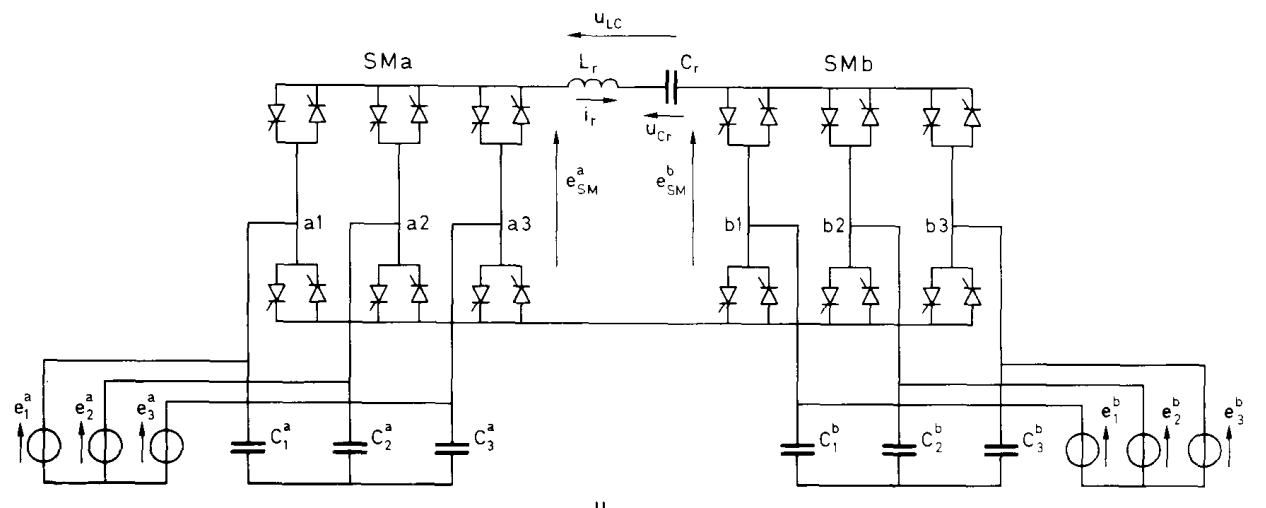

(b)

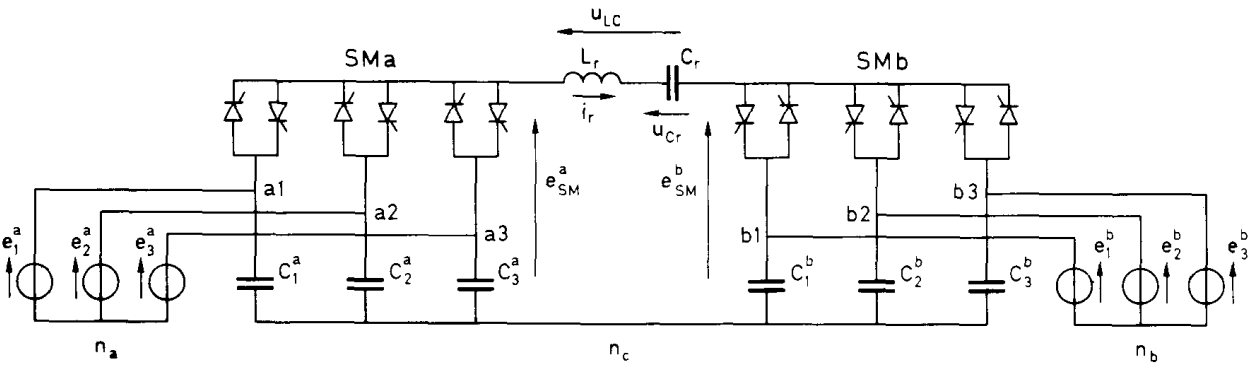

Fig. 1. Power circuit of series-resonant ac-ac converter with: (a) 24 thyristor. (b) 12 thyristors

\section{Power Circuit}

The system concerned is formally described with reference to the generalized schematic depicted in Fig. 1(b). A voltage source $e_{l}^{a}(l=1,2,3)$ is connected to the input terminals al of the switching matrix SMa via a high-frequency filter. The switching matrices SMa and SMb generate a modulated high-frequency carrier $i_{\text {r }}$ by excitation of the resonant circuit with the passive components $L_{r}$ and $C_{r}$. This carrier is distributed to the output terminals $b l(l$ $=1,2,3$ ) by switching matrix $\mathrm{SMb}$. The high-frequency content of the output current of switching matrix $\mathrm{SMb}$ is removed by the high-frequency output filter capacitors $C_{l}^{b}$. The result is a low-frequency, three-phase, sinusoidal output voltage $e_{l}^{b}$ creating a sinusoidal current through the load.

Both low pass filters and the associated controlled switching matrices SMa and SMb hold an equal rank in the system.

The polarity of the output current at one of the terminals of the switching matrix SMb depends on the process of the charging or discharging of the resonant capacitor $C_{r}$ but is independent of the polarity of the output voltages.

The series-resonant converter operates with respect to its output terminals as a converter system, which can transfer energy from the source to the load and vice versa, by reorganizing the configuration of the switches of the switching matrices SMa and SMb. This process of the selection and activation of a combination of switches is programmed by an electronic control circuit. There is a dissimilarity in the power at the output between the classical and the alternative power circuit. This can be understood by the two network topologies as shown in Fig. 2. Fig. 2(a) shows an equivalent network of the classical ac-ac series-resonant power converter with 24 thyristor switches [2]. By turning on two switches of switching matrix SMa (for example $S_{11}^{a}$ and $S_{22}^{a}$ ) the unfiltered source current $i_{s}$ for one pair of terminals (in example terminal $a 1$ and $a 2$ ) is equal to the resonant current $i_{r}$. The discussed action of the switching matrix SMa is identical to the one of switching matrix $\mathrm{SMb}$. The unselected branches in the switching network are indicated by dashed lines.

Fig. 2(b) shows the presented ac-ac series-resonant power converter with 12 thyristor switches. By turning on two switches (for example $S_{1}^{a}$ and $S_{3}^{b}$ ) the unfiltered source current $i_{s}$ for one terminal of the switching matrix SMa (this example terminal 1) is equal to the resonant current $i_{r}$

A comparison between Fig. 2(a) and (b) demonstrates a reduction in the output voltage by a factor of $\sqrt{3}$ as the difference between the line-to-line voltage and the phase voltage. The resonant current has to be increased now by a factor of $\sqrt{3}$ to obtain the same output power for both converter circuits. Similar results will be established for a comparison of a half-bridge and a full-bridge configuration of a dc-dc series-resonant converter.

\section{Control Energy Flow}

The algorithm programmed in the control circuit has to follow two principles of operation.

The first principle of operation is dominated by the control of the excitation of the series-resonant circuit. The 
(a)

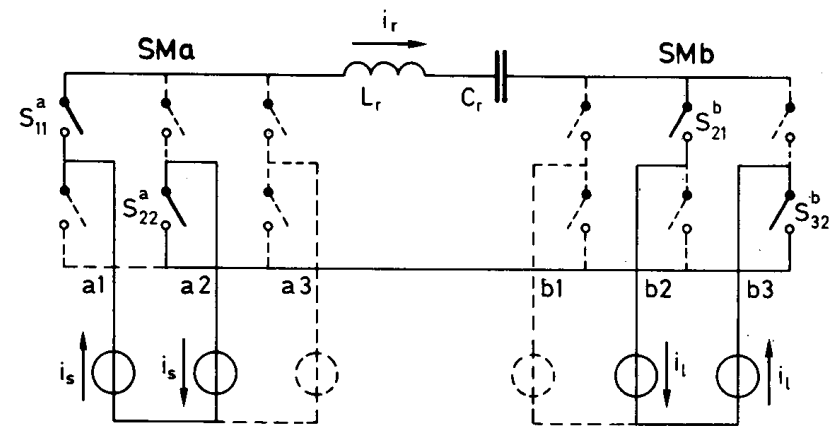

(b)

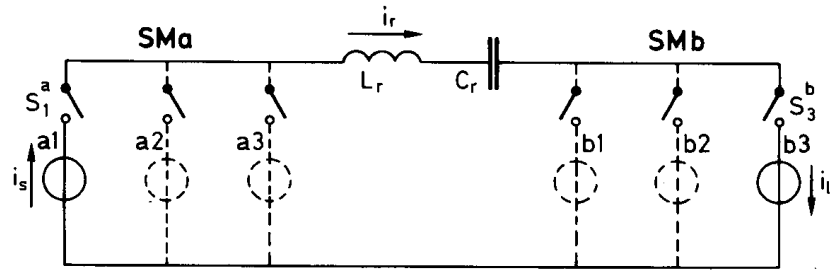

Fig. 2. Basic circuit configuration for ac-ac series-resonant converter: (a) 24 semiconductor switches, (b) 12 semiconductor switches.

resonant circuit as shown in Fig. 1, is driven by the excitation voltage $u_{L C}$ :

$$
u_{L C}=e_{S M}^{a}-e_{S M}^{b}
$$

where $e_{S M}^{a}=e_{k}^{a}, e_{S M}^{b}=e_{l}^{b}$ with $k=1,2,3$, and $l=1$, $2,3$.

To obtain a cyclic stable mode of operation for the conversion process, the net energy stored in the resonant circuit after each current pulse has to be equal. The net energy as the product of resonant current $i_{r}$ and excitation voltage $u_{L C}$, supplied to a lossless resonant circuit during a current pulse is consequently zero. The polarity of the resonant current $i_{r}$ will remain positive or negative for each individual current pulse and therefore the excitation voltage $u_{L C}$ has to change its polarity within the current pulse.

During the reverse phase the product $u_{L C} i_{r}$ has a negative value. During this time interval energy is emanated from the resonant circuit by generating an appropriate resonant current segment. During the forward phase the product $u_{L C} i_{r}$ has a positive value. During this time interval energy is boosted in the resonant circuit by generating an appropriate resonant current segment.

By changing the combination of input and output phases the polarity of the excitation voltage $u_{L C}$ may be changed.

The second principle is the described process of lowfrequency waveform generation. The control of the external waveforms of switching matrix SM2 is the primary objective of the electronic control circuit. This process follows the principles of a nonuniform and aperiodic modulation process. It has been treated in the literature [1], primarily in the context of converters which transfer and control electric energy via series-resonant circuits.

The train of bipolar resonant current pulses $i_{r}$ is distrib- uted over the three output terminals of the conversion system as indicated in Fig. 2(b). The selection of the output terminal depends on the magnitude and polarity of three error signals $\epsilon_{l}^{b}$. Each error signal is the difference between an output voltage $e_{l}^{b}$ and its sinusoidal reference voltage waveform $e_{\text {Ref } l}^{b}$ :

$$
\epsilon_{l}^{b}=e_{l}^{b}-e_{\operatorname{Ref} l}^{b} .
$$

The current pulses are converted into smoothed output voltages by three low-pass filter capacitors $C_{l}^{b}$. The use of other, passive filter elements is avoided.

Because the sum of the output voltages $e_{l}^{b}$ is bounded to a value of zero, and for this reason the sum of output currents $i_{l}^{b}$ (for a symmetrical load) is zero, the total charge on the output capacitors $C_{l}^{b}\left(C_{1}^{b}=C_{2}^{b}=C_{3}^{b}\right)$ remains neutral:

$$
\sum_{l=1}^{3} e_{l}^{b}=\sum_{l=1}^{3} Q_{l} / C_{l}^{b}=0 .
$$

Each current pulse changes the charge $Q_{l}$ on an output capacitor $C_{l}^{b}$ with a charge quantity which is small compared to its total. A great number of current pulses deliver the necessary charge $Q_{l}$ to the capacitors. This is always possible because the total charge delivered by an even number of resonant current pulses is neutral as well. The programmed control algorithm has to deliver the positive current pulses to the output phase with a negative error voltage $\epsilon_{l}^{b}$ and the negative pulses to the phase with a positive error voltage $\epsilon_{l}^{b}$.

\section{Control Algorithm}

Fig. 3 shows the flow diagram of the control algorithm of the series-resonant ac-ac converter. 


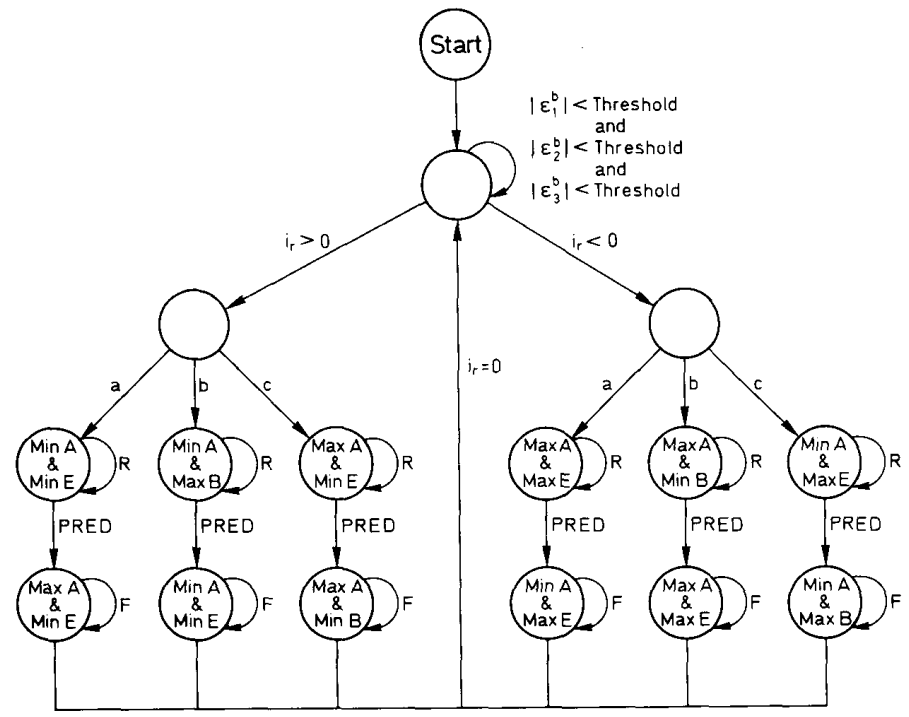

Fig. 3. Flow diagram of control algorithm.

First, the phase voltages $e_{k}^{a}$ and $e_{l}^{b}$ and the error voltages $\epsilon_{l}^{b}$ are measured and sorted with respect to their magnitudes:

$e_{\operatorname{minA}}^{a}<e_{\text {notA }}^{a}<e_{\operatorname{maxA}}^{a}:$ sorted input phase voltages, $e_{\min B}^{b}<e_{\text {notB }}^{b}<e_{\operatorname{maxB}}^{b}$ : sorted output phase voltages, $\epsilon_{\operatorname{minE}}^{b}<\epsilon_{\mathrm{notE}}^{b}<\epsilon_{\operatorname{maxE}}^{b}$ : sorted error voltages,

with voltages:

$e_{l}^{a}$ : input phase voltage connected to switcing matrix A with index $l=1,2,3$ corresponding to its phase number,

$e_{l}^{b}$ : output phase voltage connected to switching matrix $\mathrm{B}$ with index $l=1,2,3$ corresponding to its phase number,

$\epsilon_{l}^{b}$ : phase dependent error voltage connected with index $l=1,2,3$ corresponding to the index of the output phase,

The introduced subscripts are defined as

minA: subscript $\min A \in[1,2,3]$ corresponding to the phase with the most negative input voltage connected to switching matrix SMa,

$\max A: \quad$ subscript $\max A \in[1,2,3]$ corresponding to the phase with the most positive input voltage corresponding to switching matrix SMa,

notA: subscript not $\mathrm{A} \in[1,2,3]$ corresponding to the phase with the not the most positive and not the most negative input voltage connected to switching matrix SMa,

$\min B$ : subscript $\min B \in[1,2,3]$ corresponding to the phase with the most negative output voltage connected to switching matrix $\mathrm{SMb}$,

$\operatorname{maxB}$ : subscript $\max B \in[1,2,3]$ corresponding to the phase with the most positive output voltage corresponding to switching matrix $\mathrm{SMb}$,
notB: subscript notB $\in[1,2,3]$ corresponding to the phase with the not the most positive and not the most negative output voltage connected to switching matrix $\mathrm{SMb}$,

minE: subscript $\min E \in[1,2,3]$ corresponding to the phase with the most negative error voltage related to switching matrix $\mathrm{SMb}$,

maxE: subscript maxE $\in[1,2,3]$ corresponding to the phase with the most positive error voltage related to switching matrix $\mathrm{SMb}$,

notE: subscript notE $\in[1,2,3]$ corresponding to the phase with the not the most positive and not the most negative error voltage related to switching matrix $\mathrm{SMb}$.

The switching algorithm is independent of the polarity of the resonant current and consequently equal for each pulse. The positive arm will be explained in the following text. The negative arm is of the same structure. Two combinations, of an input and an output voltage, have to be selected to generate the necessary positive and negative excitation voltage $u_{L C}$ to operate in the Reverse and Forward modes. These two excitation voltages are built from at least three voltage sources connected to the converter terminals. One commutation is accomplished for each resonant current pulse. One of these phase voltages is related to an error voltage $\epsilon_{\min }^{b}$ because the positive current pulse will decrease a negative error voltage to obey the generation process of the output waveform. The selection of the additional two phases depends on the magnitude of the phase voltage $e_{\min }^{b}$ compared to $e_{\operatorname{minA}}^{a}$ and $e_{\operatorname{maxA}}^{a}$. Three situations can be illustrated:

1) $e_{\operatorname{minA}}^{a}<e_{\operatorname{minE}}^{b}<e_{\operatorname{maxA}}^{a}$ : step-down mode,

2) $e_{\operatorname{minB}}^{b}<e_{\operatorname{maxA}}^{a}<e_{\min }^{b}$ : step-up mode,

3) $e_{\operatorname{minE}}^{b}<e_{\operatorname{minA}}^{a}<e_{\operatorname{maxB}}^{b}$ : step-up mode. 


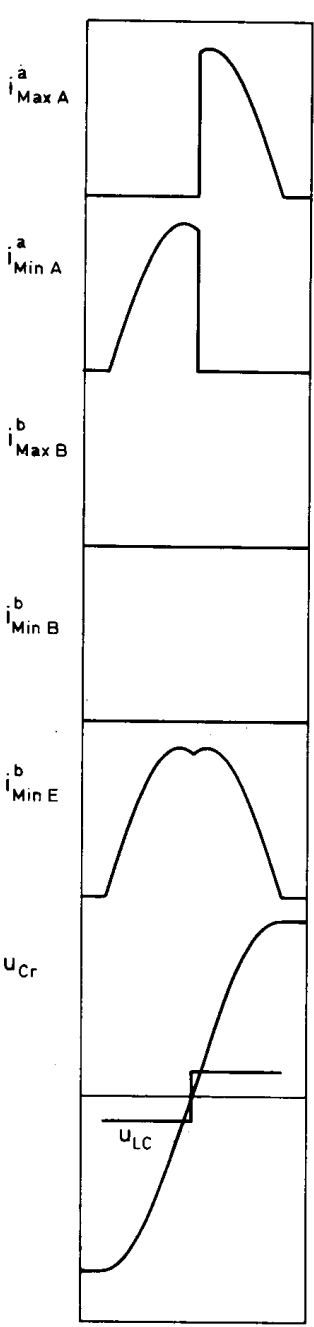

(a)

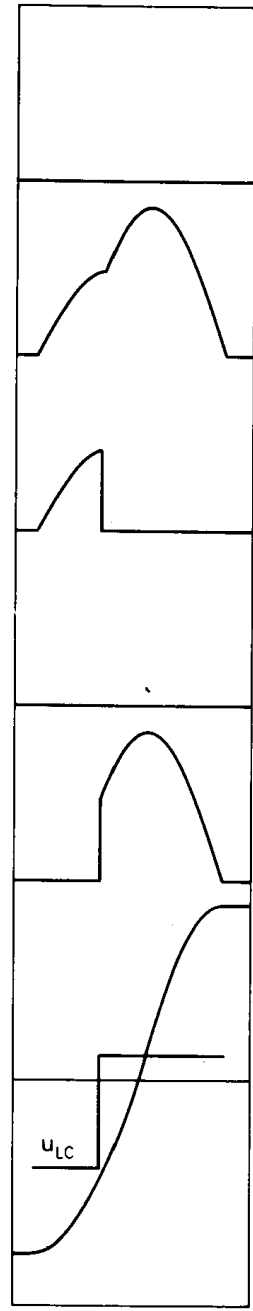

(b)

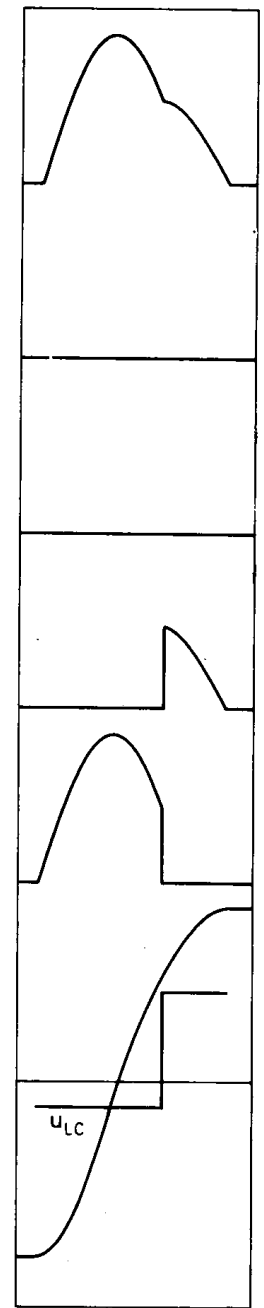

(c)

Fig. 4. Typical waveforms of resonant current $i_{r}$, excitation voltage $u_{l C}$, and resonant capacitor voltage $u_{C r}$, for various modes of operation: (a) Step-down mode: $e_{\operatorname{minA}}^{a}<e_{\operatorname{minE}}^{b}<e_{\operatorname{maxA}}^{a}$. (b) Step-up mode: $e_{\operatorname{minB}}^{b}<e_{\operatorname{maxA}}^{a}<e_{\operatorname{minE}}^{b}$. (c) Step-up mode: $e_{\operatorname{minE}}^{b}<e_{\min \mathrm{A}}^{a}<e_{\operatorname{maxB}}^{b}$

$e_{\operatorname{minE}}^{b}$ is the phase voltage of the output phase corresponding to an error voltage $\epsilon_{\operatorname{minE}}^{b}$. Those three situations supply different combinations as indicated through the waveforms of Fig. 4.

1) Step-down mode: $e_{\operatorname{minA}}^{a}<e_{\min E}^{b}<e_{\operatorname{maxA}}^{a}$. During the first resonant current segment (reverse phase), the excitation voltage $u_{L C}$ is equal to

$$
u_{\mathrm{LCR}}=e_{\operatorname{minA}}^{a}-e_{\operatorname{minE}}^{b}<0
$$

The energy in the resonant network will be boosted during the second current segment (Forward phase) by the excitation voltage $u_{L C}$ :

$$
u_{\mathrm{LCF}}=e_{\operatorname{maxA}}^{a}-e_{\operatorname{minE}}^{b}>0
$$

This step-down mode is ideal because the complete current pulse will decrease the error voltage $\epsilon_{\operatorname{minE}}^{b}$.

2) Step-up mode: $e_{\operatorname{minB}}^{b}<e_{\operatorname{maxA}}^{a}<e_{\operatorname{minE}}^{b}$. During the first resonant current segment, the excitation voltage $u_{L C}$ is equal to

$$
u_{\mathrm{LCR}}=e_{\operatorname{maxA}}^{a}-e_{\operatorname{minE}}^{b}<0
$$

The energy in the resonant network will be boosted during the second current segment by the excitation voltage $u_{L C}$ :

$$
u_{\mathrm{LCF}}=e_{\operatorname{maxA}}^{a}-e_{\operatorname{minB}}^{b}>0
$$

The excitation voltage $u_{\mathrm{LCF}}$ is continually positive because $e_{\operatorname{maxA}}^{a}$ is positive $e_{\operatorname{minB}}^{b}$ is always negative. This stepup mode is less effective than the step-down mode, be- 
cause the error voltage $\epsilon_{\min }^{b}$ will be decreased only during the resonant segment of the Reverse phase.

3) Step-up mode: $e_{\operatorname{minE}}^{b}<e_{\operatorname{minA}}^{a}<e_{\operatorname{maxB}}^{b}$. During the first resonant current segment, the excitation voltage $u_{L C}$ is equal to:

$$
u_{\mathrm{LCR}}=e_{\operatorname{minA}}^{a}-e_{\operatorname{maxB}}^{b}<0
$$

The excitation voltage $u_{\mathrm{LCR}}$ is always negative because $e_{\operatorname{minA}}^{a}$ is negative and $e_{\operatorname{maxB}}^{b}$ always positive. The energy in the resonant network will be boosted during the second current segment by

$$
u_{\mathrm{LCF}}=e_{\operatorname{minA}}^{a}-e_{\operatorname{minE}}^{b}>0
$$

This step-up mode is less effective than the step-down mode, because the error voltage $\epsilon_{\min }^{b}$ will be decreased only during the resonant current segment of the forward phase. The switching algorithm has some more features as explained in Fig. 3.

(a) The resonant current pulse will only be started if there is at least one error voltage $\epsilon_{i}^{b}$ with an amplitude larger than a specific threshold value.

(b) The generated resonant current pulse will be positive if the initial resonant capacitor voltage $u_{C r}$ is negative and vice versa.

(c) The switching time between the Reverse and the forward current segment is under the control of an electronic circuit indicated as the predictor. This circuit calculates the stored energy in the resonant network at the end of the resonant current pulse.

(d) The algorithm is reset to its initial status if a detection circuit for the resonant current observed a zerocrossing of this current.

\section{InTERACTION CONVERTER, LoAd AND SOURCE}

The character of the load: capacitive, inductive or dissipative, does influence the output currents $i_{1}^{b}, i_{2}^{b}$ and $i_{3}^{b}$ but not the output voltages $e_{1}^{b}, e_{2}^{b}$ and $e_{3}^{b}$ as long as the converter is not overloaded.

For a symmetrical load network in star configuration the voltage of the neutral $n_{b}$ of the load will be at zero. The output currents are symmetrical because the output voltages are symmetrical while the sum of the output currents is zero.

The voltage of the neutral of the load is at zero and may therefore be connected to the neutral of the converter without current flowing through this link. Only a small asymmetry between the output voltages caused by the high-frequency ripple voltages generates a low high-frequency current through the link between the neutral of converter $n_{c}$ and the neutral of load $n_{b}$ as measured and recorded in Figs. 5 and 6 . The parameters of the converter for the experiments are

$$
\begin{aligned}
L_{r} & =19 \mu \mathrm{H}, \quad C_{r}=2.7 \mu \mathrm{F}, \\
C_{l}^{a} & =C_{l}^{b}=100 \mu \mathrm{F}(l=1,2,3), \\
R_{l} & =20 \Omega,
\end{aligned}
$$

$$
\begin{aligned}
& e_{\text {max }}^{a}=100 \mathrm{~V}, 50 \mathrm{~Hz}, \\
& e_{\text {max }}^{b}=100 \mathrm{~V}, 50 \mathrm{~Hz} .
\end{aligned}
$$

The source must be able to generate and accept electric power. The neutral $n_{a}$ of a symmetrical source can be connected to the neutral $n_{c}$ of the converter. Each voltage source $e_{1}^{a}, e_{2}^{a}$ and $e_{3}^{a}$ will charge the filter capacitors $C_{1}^{a}$, $C_{2}^{a}$ and $C_{3}^{a}$ to match their voltage. Only high-frequency components of the souce currents flows through the link between $n_{a}$ and $n_{c}$. The amplitude of this high-frequency current depends on the source impedances and is recorded in Fig. 7.

In the case of an asymmetrical load the individual output currents are different. The influence of an asymmetrical load on the output voltages depends on the asymmetry of the load and whether the neutral of converter and load are tied together. Three different modes can be distinguished now:

1) The particular neutrals of converter $n_{c}$ and load $n_{b}$ are floating. Therefore the total of the output currents is equal to zero (Kirchoff's law: $i_{1}^{b}+i_{2}^{b}+$ $i_{3}^{b}=0$ ) even for an asymmetrical load. The total charge on the output capacitors $\left(C_{1}^{b}, C_{2}^{b}\right.$ and $\left.C_{3}^{b}\right)$ is maintained and the distribution of the charge over the capacitors remains symmetrical, as do the output voltages.

2) The neutral of the load $n_{b}$ remains at zero when it is connected to the neutral of the converter $n_{c}$. The sum of the output currents will no longer be equal to zero $\left(i_{1}^{b}+i_{2}^{b}+i_{3}^{b} \neq 0\right)$. The result is a low-frequency current running through the connection between the neutrals. Therefore the total charge on the output capacitors $C_{1}^{b}, C_{2}^{b}$ and $C_{3}^{b}$ differs now from zero. The series-resonant circuit cannot supply a low-frequency compensation current. A symmetrical division of the charge across the output capacitors is not possible and the output voltages $e_{1}^{b}, e_{2}^{b}$ and $e_{3}^{b}$ become asymmetrical.

3) The output voltages $e_{4}^{b}, e_{5}^{b}$ and $e_{6}^{b}$ will be asymmetrical if the output current is equal to the maximum current which the converter can supply. This happens when an asymmetrical load impedance becomes close to zero (a short circuit between two terminals). Two output phase-voltages are equal in magnitude. The third output phase-voltage has an opposite sign with an amplitude of a factor of two.

\section{Control Electronics}

Fig. 8 depicts a block diagram of the control electronics of the series-resonant power converter. This figure is the result of the flow diagram presented in Fig. 3. The analog voltage and current waveforms are located in the middle of the block diagram. At the left of this diagram there are four test circuits which produce signals for the control circuit. At the right of this diagram there are the selector circuits for activating the proper thyristors. 


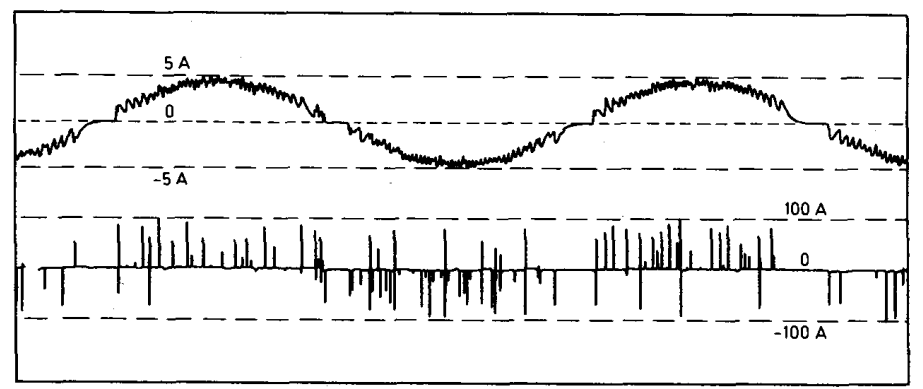

Fig. 5. Measurement of output current with resistive load (time $40 \mathrm{~ms}$ ) Upper trace: load current $i_{l}^{b}$. Lower trace: switch current $i_{S_{i}^{t}}$.

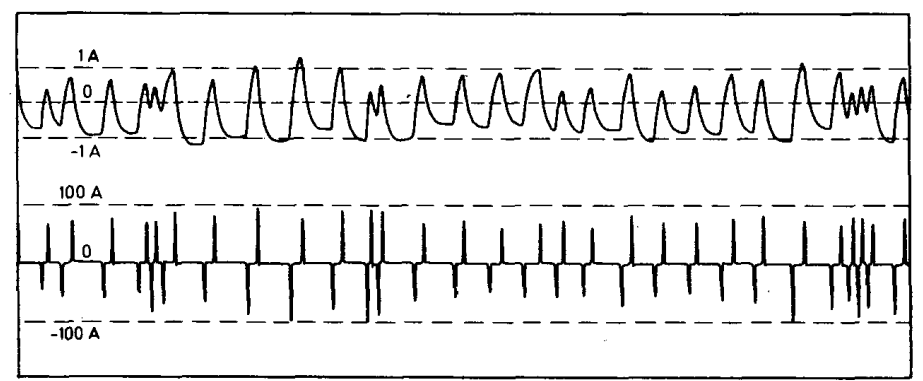

Fig. 6. Measurement of current through link between $n_{c}$ and $n_{b}$ (time $10 \mathrm{~ms}$ ). Upper trace: current through the link. Lower trace: resonant current $i_{r}$.

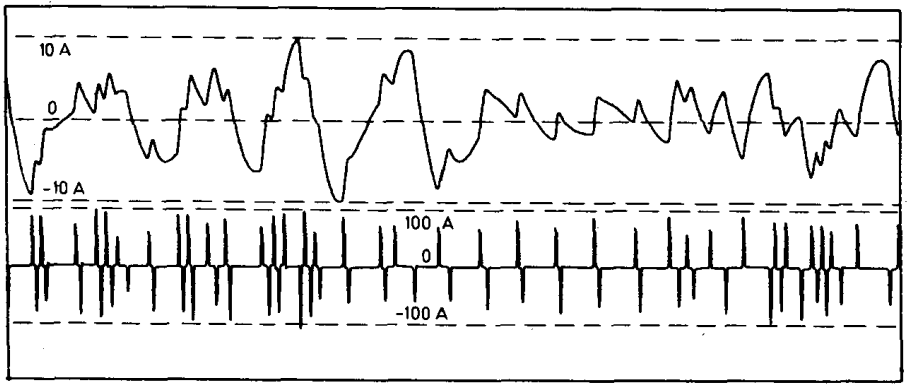

Fig. 7. Measurement of current through line between $n_{a}$ and $n_{c}$ (time $10 \mathrm{~ms}$ ). Upper trace: current through the link. Lower trace: resonant current $i_{r}$.

The $u_{C r}$ POLARITY detector detects the polarity of the resonant capacitor voltage $u_{C r}$. For a positive voltage, the capacitor $C_{r}$ will be discharged and the next current pulse $i_{r}$ will be consequently negative. For a negative voltage, the capacitor $C_{r}$ will be charged and the next current pulse $i_{r}$ will be consequently positive.

The point in time where internal signals will be changed is locked to the zero crossing of the resonant current $i_{r}$ at the end of the resonant current pulse by the ZERO CURRENT DETECTOR. The CONTROL CIRCUIT can start a new current pulse when the resonant current is zero.

The $u_{L C}$ POLARITY DETECTOR checks at the beginning of each current pulse whether the excitation voltage $u_{L C}$ has the proper polarity. Control of the electric energy stored in the resonant capacitor $C_{r}$ is necessary to obtain an uninterrupted series-resonant oscillation [5]. The PREDICTOR controls the excitation of the series-resonant circuit from pulse to pulse in a predictive way on extrapolating the signals $i_{r}, u_{C r}$ and $u_{L C}$.

This control has two purposes:

1) to limit the momentary amplitude of the resonant capacitor voltage $u_{C r}$ from pulse to pulse to avoid excessive component stresses,

2) to satisfy the conditions of a continuous oscillation for each half period independently of the waveform generated at the output.

The analogue signals corresponding to $e_{k}^{a}$ and $e_{l}^{b}$, are the input waveforms of the $u_{L C}$ POLARITY DETECTOR and the PREDICTOR. The $k$ and $l$ value is selected by the 


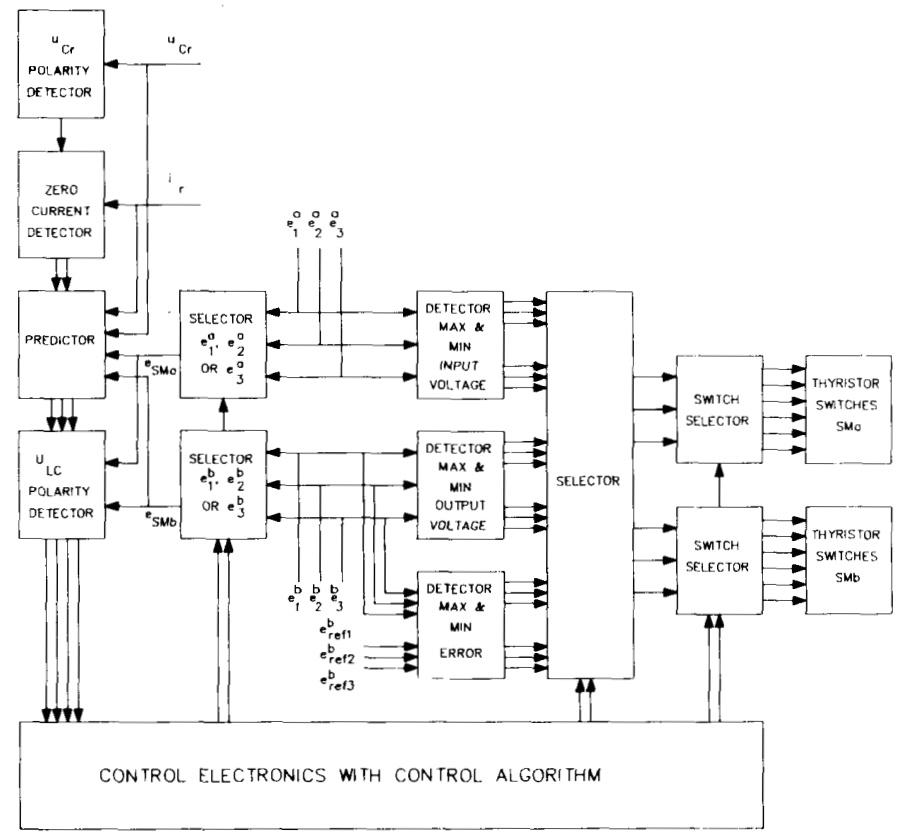

Fig. 8. Block diagram of control electronics.

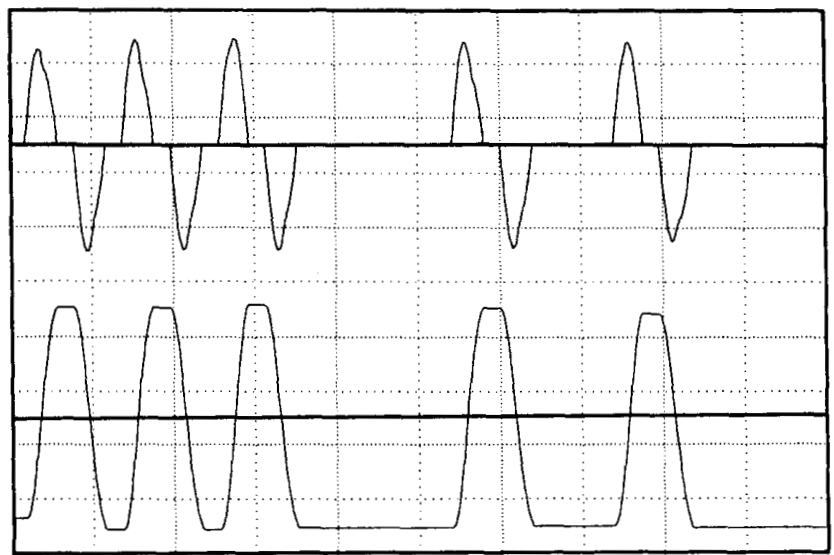

Fig. 9. Results of computer simulation [time $1 \mathrm{~ms}$ ]: Upper trace: resonant current $i$, [50 A/div]. Lower trace: resonant capacitor voltage $u_{C},[150 \mathrm{~V} / \mathrm{div}]$

SELECTOR $e_{1}^{a}, e_{2}^{a}$ or $e_{3}^{a}$ and the SELECTOR $e_{2}^{b}, e_{2}^{b}$ or $e_{3}^{b}$. The switch selector is responsible for the selection process of the active switching elements in both switching matrices, depending on the conversion ratio of the power converter.

\section{Computer Simulation and Experimental Results}

The 12-thyristor converter was simulated by a computer simulation program [3]. The results of simulation are indicated in Figs. 9 and 10, confirming the characteristics of this class of resonant ac-ac converters. The pa- rameters of the converter for the simulation were:

$$
\begin{aligned}
L_{r} & =19 \mu \mathrm{H}, \quad C_{R}=2.7 \mu \mathrm{F}, \\
C_{l}^{a} & =C_{l}^{b}=100 \mu \mathrm{F}(l=1,2,3), \\
e_{\text {max }}^{a} & =50 \mathrm{~V}, 60 \mathrm{~Hz}, \\
e_{\text {mmax }}^{b} & =100 \mathrm{~V}, 50 \mathrm{~Hz} .
\end{aligned}
$$

An experimental series-resonant converter with twelve thyristors was designed and constructed. Existing components and circuits were used to demonstrate a principle 


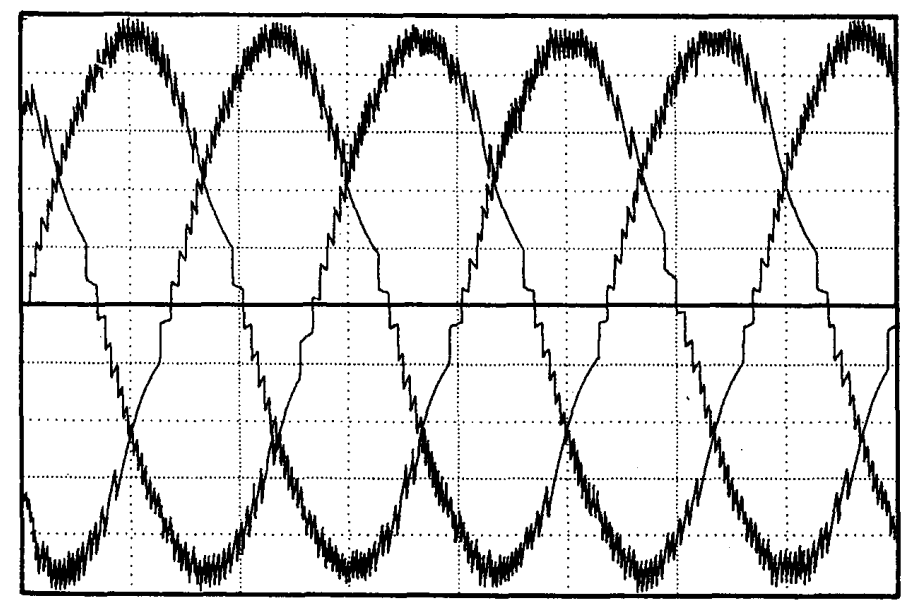

Fig. 10. Results of computer simulation [time $40 \mathrm{~ms}$ ]: Output voltages $e_{1}^{b}, e_{2}^{b}$ and $e_{3}^{b}[20 \mathrm{~V} / \mathrm{div}]$.

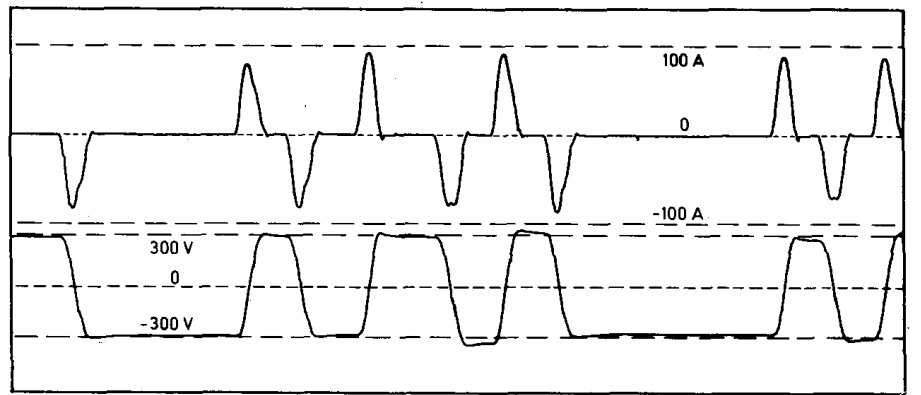

Fig. 11. Measurement of $i_{r}$ and $u_{C r}$ [time $1 \mathrm{~ms}$ ]. Upper trace: resonant current $i_{r}$. Lower trace: capacitor voltage $u_{C r}$.

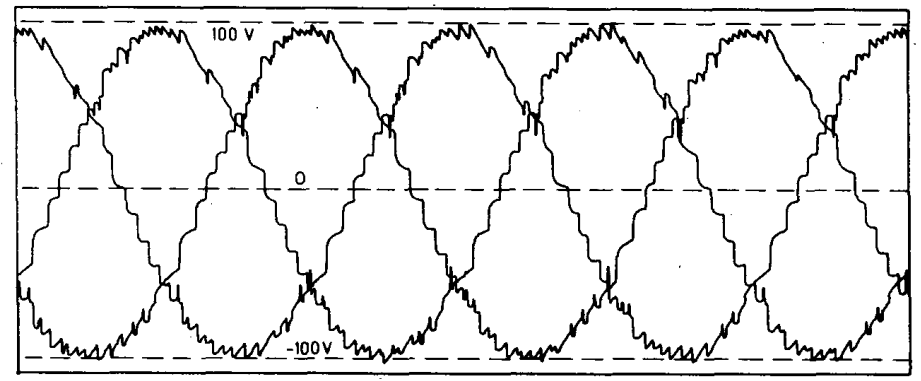

Fig. 12. Measurement of output voitages $e_{1}^{b}, e_{2}^{b}$ and $e_{3}^{h}$ (time of measurement $40 \mathrm{~ms}$ ).

rather than to optimize the system. Figs. 11, 12, and 13 were taken with a digital recorder (resolution 8 bits, sample rate $20 \mathrm{~ns}$ ) and copied on a $X-Y$ plotter with the following conditions shown in Table I.

Fig. 11 shows the aperiodic waveforms of the resonant current $i_{r}$ and the resonant capacitor voltage $u_{C r}$. The amplitude of the resonant capacitor voltage appears to be constant.

The output voltages are recorded in Fig. 12 for an output frequency of $50 \mathrm{~Hz}$.

Finally, Fig. 13 shows the current $i_{l}^{a}$ at the input matrix SMa. This current is delivered during the time interval that a particular source voltage is maximal. Other tests were carried out for different values of the output current and voltage and a range for the fundamental frequency $f_{l}^{b}$ of a three terminal, sinusoidal output voltage of $0<$ $f_{l}^{b}<100 \mathrm{~Hz}$. The system was tested with respect to:

(a) functional integrity under adverse conditions of operation,

(b) output voltage control,

(c) efficiency of the conversion process.

\section{Conclusion}

The series-resonant ac-ac converter with twelve thyristor switches has the following advantages:

1) The converter with a reduced number of bipolar switches, has only 12 thyristors and consequently 12 gate pulse amplifiers: less than the 24-thyristors 


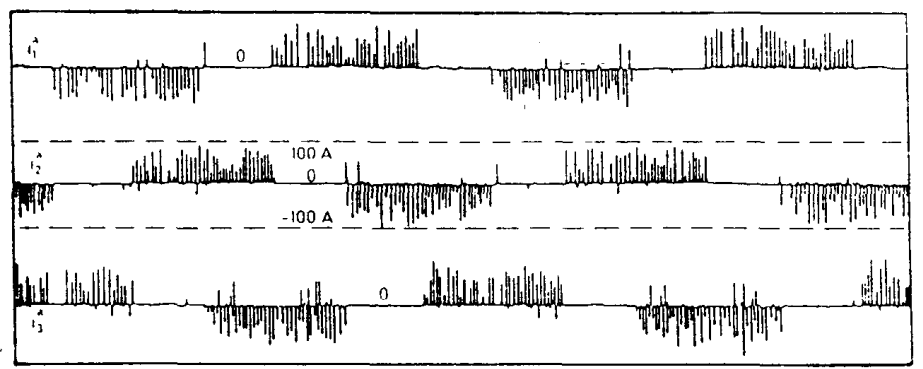

Fig. 13. Measurement of input currents $i_{1}^{t \prime}, i_{2}^{t t}$ and $i_{3}^{u}$ [time of measurement $40 \mathrm{~ms}$ ].

TABLE 1

\begin{tabular}{|c|c|c|c|c|}
\hline & Source & & Load & $\begin{array}{l}\text { Resonant } \\
\text { Converter }\end{array}$ \\
\hline$P^{\prime \prime}$ & $=2.046 \mathrm{~kW}$ & $P^{b}$ & $=1.677 \mathrm{~kW}$ & $P_{1 \mathrm{l}}=0.390 \mathrm{~kW}$ \\
\hline$f_{l}^{a}$ & $=50 \mathrm{~Hz}$ & $f_{i}^{b}$ & $=50 \mathrm{~Hz}$ & $f_{\text {Fulat }}=9.09 \mathrm{kHz}$ \\
\hline$e_{\mathrm{RMS}}^{a}$ & $=70.7 \mathrm{~V}$ & $e_{\text {IRMS }}^{i_{3}}$ & $=51.8 \mathrm{~V}$ & dist $=1.3 \%$ \\
\hline$i_{\text {RRMS }}^{A}$ & $=9.65 \mathrm{~A}$ & $i_{\text {iRMS }}^{h}$ & $=10.8 \mathrm{~A}$ & $i_{\max }=90 \mathrm{~A}$ \\
\hline
\end{tabular}

converter. This results in lower costs and a less complex electronic control and protection system.

2) The input voltage of the 12-thyristor converter equals the phase voltage of a 3-terminal source voltage, whle the 24-thyristor converter applies the lineto-line voltage. It implies less stress on the components of the converter when the source voltages are equal, but it is also possible to apply to the 12-thyristor converter a $\sqrt{3}$ times higher source voltage with the same stress on the components.

3) The 12-thyristor converter which distributes the same amount of power with the same source voltages as the 24-thyristor converter, needs a higher resonant current of a factor of $\sqrt{3}$ times. for the 12-thyristor converter the current passes only two thyristors, while in the more complex circuit four thyristors are passed. The losses in the thyristors are lowered but the losses in the resonant inductor are increased. Redimensioning of the resonant inductor for less losses gives the 12-thyristor converter a higher degree of efficiency.

4) Grounding of the individual neutrals of the capacitor banks, the load and the converter, is possible. It is noted that this will not be feasible for a 24-thyristor converter.

5) The sum of the output voltages is forced to be zero for the 12-thyristor converter. An unbalanced voltage system may lead to saturation of transformers and electrical machines.

The series-resonant ac-ac converter with 12 thyristor switches will have the following disadvantages:

1) the multi-terminal voltage supply has to contain at least a positive and a negative voltage source.

2) the power factor of the source current can not yet be controlled [5].

In comparison with the whole group of three-phase acac converters, this ac-ac series-resonant converter with the minimal number of 12 thyristor switches (one unipolar switch for each terminal and current polarity makes a total of twelve switches) demonstrates the characteristics of:

low cost

high efficiency,

low distortion of the output waveforms, prevention of excessive stress on components, fast system response.

Electrical characteristics were experimentally verified and have been proven by simulation.

\section{REFERENCES}

[1] F. C. Schwarz, "An improved method of resonant current pulse modulation for power converters," IEEE Trans. Ind. Electron. Contr. Instrum., vol. IECI-23. no. 2. pp. 133-141. May 1976.

[2] J. B. Klaassens, "DC to ac series-resonant converter system with high internal frequency generating multiterminal ac waveforms for multikilowatt power levels," IEEE Trans. Power Electron., vol. PE-2, no. 3. pp. 247-256. July 1987.

[3] J. B. Klaassens, J. van Duivenbode, "Series-resonant energy conversion with multi-segment current waveforms for bipolar energy flow," in IEEE Power Electronics Specialists Conf. Rec., Kyoto, Japan. Apr. 11-14. 1988 . pp. 599-608.

[4] J. B. Klaassens, "DC to ac series-resonant converter system with high internal frequency generating synthesized waveforms for multikilowatt power levels." in IEEE Poner Electronics Specialists Conf. Rec.. Gaithersburg. MO. June 1984. pp. 99-110, and IEEE Trans. Power Electron., vol. PE-1, no. I. Jan. 1986, pp. 9-20.

[5] J. B. Klaassens, E. J. F. M. Smits, "Series-resonant ac-power interlace with an optimal power factor and enhanced conversion ratio," IEEE Trans. Power Electron.. PE-3, no. 3, pp. 335-343, July 1988.

[6] H. K. Lauw, J. B. Klaassens. N. G. Butler, and D. B. Seely, "Variable-speed generation with the Series-Resonant Converter," in IEEE Power Engineering Society Winter Meeting Rec.. New York, February $1-5.1988$

[7] H. Huisman. "A three-terminal to three-terminal series-resonant power converter with optimal input current waveforms, Part l: Control Strategy. Part II: Application and Results," IEEE Trans. Ind. Electron. IE-35. no. 2. pp. 263-277, May 1988.

[8] T. A. Lipo, "Recent Progress in the Development of Solid-State ACMotor drives, "IEEE Trans. Power Electronics, PE-3, no. 2, pp. 105110. Apr. 1988.

J. Ben Klaassens, for a photograph and biography see this issue, p. 337 .

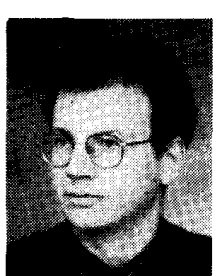

Freddie G. de Beer was born in Almelo. The Netherlands on August 12, 1965. He received the M.S. degree in electrical engineering from the Delft University of Technology, Delft, The Netherlands in 1989 . His master thesis was on the field of an ac-ac series-resonant converter with reduced number of thyristors. He is currently working on his Ph.D. thesis in power electronics and motor control at the same University. 